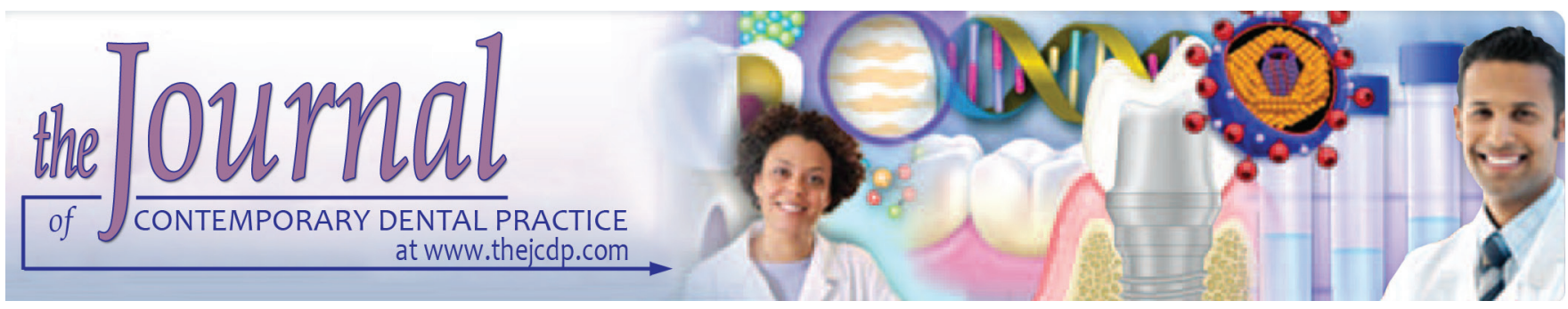

\title{
Gingival Zenith Positions and Levels of Maxillary Anterior Dentition in Cases of Bimaxillary Protrusion: A Morphometric Analysis
}

${ }^{1}$ Snigdha Gowd, ${ }^{2} \mathrm{~T}$ Shankar, ${ }^{3}$ Suravi Chatterjee, ${ }^{4}$ Pritam Mohanty, ${ }^{5}$ Nivedita Sahoo, ${ }^{6}$ Srinivas Baratam

\begin{abstract}
Aim: To investigate the two clinical parameters, such as gingival zenith positions (GZPs) and gingival zenith levels (GZLs), of maxillary anterior dentition in bimaxillary protrusion cases and collate it with severiety of crown inclination.
\end{abstract}

Materials and methods: Gingival zenith position and GZL in 40 healthy patients ( 29 females and 11 males) with an average age of 21.5 years were assessed. Inclusion criteria involved absence of periodontal diseases, Angle's class I molar relationship, and upper anterior proclination within 25 to $45^{\circ}$ based on Steiner's analysis; exclusion criteria included spacing, crowding, anterior restoration and teeth with incisor attrition or rotation.

The GZP was evaluated using digital calipers from voxelbased morphometry (VBM), and GZL was assessed from the tangent drawn from GZP of central incisor and canines to the linear vertical distance of GZP of lateral incisor.

Results: All the central incisors showed a GZP distal to VBM with a mean average of $1 \mathrm{~mm}$. Severe proclination between 40 and $45^{\circ}$ showed a statistically significant variation. Lateral incisors displayed a mean of $0.5 \mathrm{~mm}$ deviation of GZP from the vertically bisected midline. In $80 \%$ of canine population, GZP was centralized.

Conclusion: We conclude that the degree of proclination of maxillary anterior dentition was correlated to the gingival contour in bimaxillary cases. The investigation revealed that there is a variation in the location of GZP as the severity of proclination increases.

${ }^{1,3-6}$ Department of Orthodontics, Kalinga Institute of Dental Sciences, KIIT University, Bhubaneswar, Odisha, India

${ }^{2}$ Department of Prosthodontics, Kalinga Institute of Dental Sciences, KIIT University, Bhubaneswar, Odisha, India

Corresponding Author: Suravi Chatterjee, Department of Orthodontics, Kalinga Institute of Dental Sciences, KIIT University Bhubaneswar, Odisha, India, Phone: +918339034769, e-mail: riya.junk@gmail.com
Clinical significance: This study highlights the importance of microesthetics in fixed orthodontic treatment. The gingival contour should be unaltered while retraction during management of bimaxillary protrusion.

Keywords: Gingival zenith level, Gingival zenith position, Inclination, Maxillary anterior dentition, Tipping.

How to cite this article: Gowd S, Shankar T, Chatterjee S, Mohanty P, Sahoo N, Baratam S. Gingival Zenith Positions and Levels of Maxillary Anterior Dentition in Cases of Bimaxillary Protrusion: A Morphometric Analysis. J Contemp Dent Pract 2017;18(8):700-704.

Source of Support: Nil

Conflict of Interest: None

\section{INTRODUCTION}

Assembling the tooth within the frame of gingival margins plays a symbolic role in smile designing and has a tremendous impact on the overall facial and dentogingival esthetics, and as orthodontists, it is our duty to harmoniously restore and balance the same. The term bimaxillary protrusion was first used by Calvin Case. ${ }^{1}$ He describes bimaxillary protrusion as a condition where the entire dentures of both the jaws are protruded in relation to mandible and other bones of the skull. It is seen commonly in African-American ${ }^{2-5}$ and Asian ${ }^{6-8}$ populations. It is usually combined with lip incompetence, gummy smile, mentalis strain, and anterior open bite. As of the negative perspicuity of protrusive dentition and lips in various cultures, numerous patients with bimaxillary protrusion seek orthodontic care to decrease this procumbency. Facial esthetics, therefore, is the primary concern of these patients. Understanding of the ideal gingival topography has critical importance for the multidisciplinary diagnosis and treatment in the anterior dentition. 
The construct of an ideal smile requires assessment and proper analysis of the face, lips, gingival tissues, and teeth and an appraisal of conjointly how they appear. Hence, assessing all the components of a balanced smile is of paramount importance in cases of bimaxillary protrusion. Gingival heath comes first among the esthetic objective while treatment planning is under consideration. It is crucial to contemplate gingival morphology and contour. ${ }^{9}$ The gingival morphology is said to have knife-edged tightly adapted gingival margin, interdental grooves, and cone-shaped interdental papilla. ${ }^{10}$ Gingival zenith position (GZP) is defined as the most apical position of the cervical tooth margin. It is located slightly distal to the vertical line drawn down the center of the tooth (Figs 1 and 2). Establishing, the proper location of zenith points is a critical step in alteration of mesial and distal dimensions. Moreover, the zenith on the maxillary central incisors and canines was described as at the same height, and that on the lateral incisors was described as slightly coronal. ${ }^{11,12}$

One significant feature of gingival morphology along with gingival zenith is the gingival zenith level (GZL), which is defined as the line joining the tangents of the gingival zeniths of the central incisor and canine. Any kind of gingival asymmetry in bimaxillary protrusion will lead to visual stress and imbalance accentuating the already protrusive appearance. It is mandatory to correctly position the zenith following therapeutic manipulation because it can remarkably dominate the emergence profile and axial inclination of the teeth by readapting the line angle position of the long axis of the emergence of the crown from the gingiva. ${ }^{13,14}$

Despite its critical importance, to the best of our knowledge, morphometric evaluation of GZP and GZL in maxillary anterior dentition with increased crown angulation is not available. Thus, the intention of our research was to assess and establish the two clinical parameters GZP and GZL in bimaxillary protrusion and collate with a degree of crown inclination.

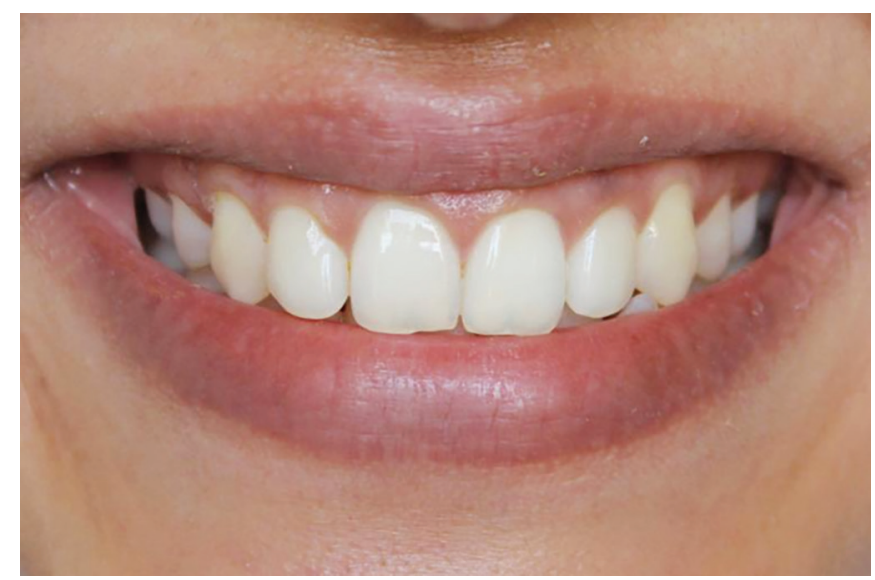

Fig. 1: Ideal gingival architecture with knife-edge margins

\section{MATERIALS AND METHODS}

\section{Study Population and Experimental Design}

The study was directed in conformance with the Helsinki declaration of 1975, as revised in 2000, and after institutional research and ethical committee clearance. Informed consent was received from all the patients participating in the study. The experimental sample consisted of 40 adult patients (11 males and 29 females), 20 to 23 years of age (mean, 20.8 years), with healthy gingival tissue and good systemic health.

Inclusion criteria containing population with nonrestored maxillary anterior teeth, teeth with no anterior crowding, no signs of incisal attrition, gingival recession or passive eruption, no previous history of orthodontic treatment, or where fixed or removable orthodontic treatment has not commenced were included in the study. Anterior teeth with mild rotations were excluded from the sample.

\section{Clinical Data Collection}

To establish the relative protrusion of the dentition, the angle between the long axis of the maxillary central incisors and the nasion/A-point line was measured in degrees, along with the distance between the mandibular central incisor tip and the nasion/A-point line in millimeters. These measurements were taken from Steiner analysis.

The millimeter distance establishes how prominent the incisors are relative to the supporting bone, while the inclination indicates whether the teeth have been tipped to their position or have moved there bodily. ${ }^{15}$ Patient with proclination of 25 to $45^{\circ}$ fulfilling all the inclusion and radiological criteria were clinically assessed, and silicone impressions of maxillary dentition were taken in the stock tray and poured with orthokal.

\section{Morphometric Data Analysis}

Reference lines were drawn on the stone caste as per $\mathrm{Chu}$ et $\mathrm{al}^{16}$ (Fig. 2). The cast was evaluated by an operator

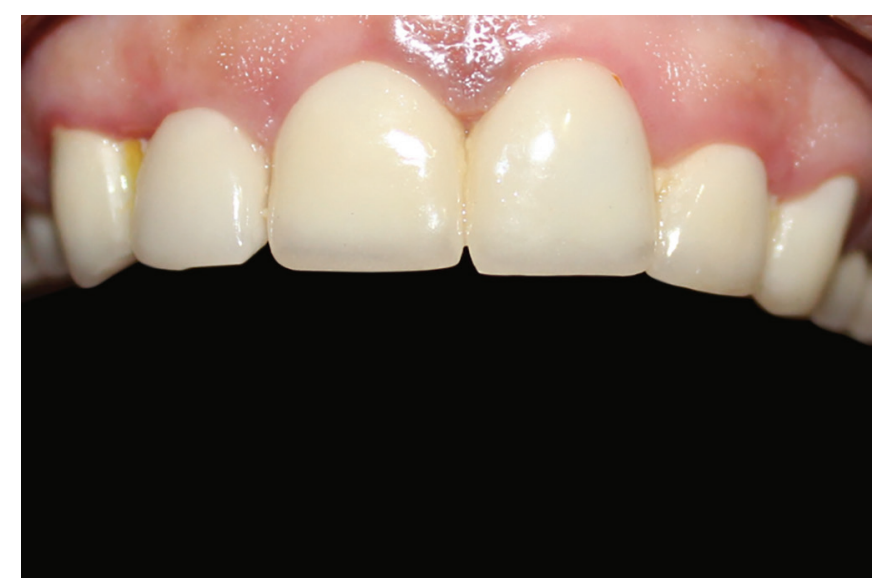

Fig. 2: Disproportionate gingival margin in bimaxillary protrusion 


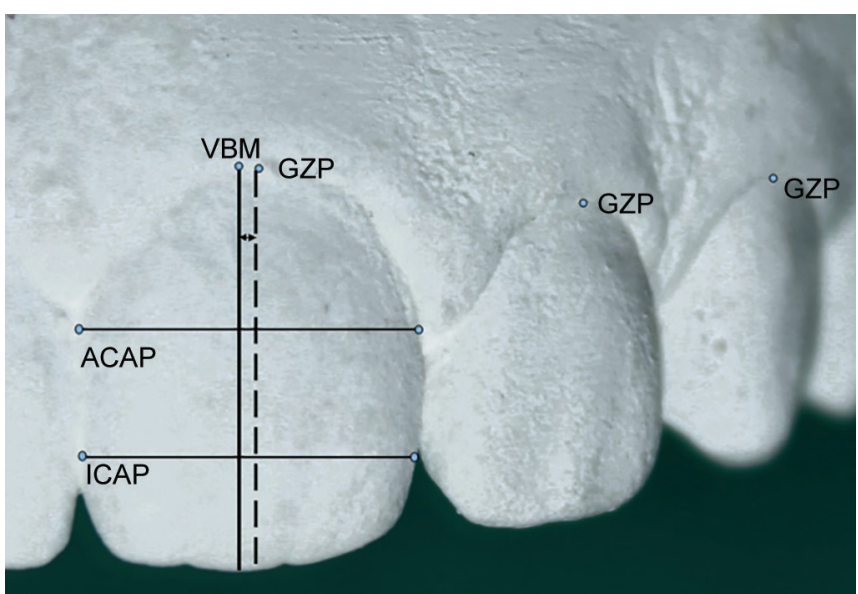

Fig. 3: Morphometric measurement of GZPs; ACAP: Apical contact area position; ICAP: Incisal contact area position; VBM: Vertical bisected line

using 2.5 magnification optical loupes. Six inch digital caliper (i.e., graduation: $0.01 \mathrm{~mm}$, accuracy: $0.02 \mathrm{~mm}$, repeatability: $0.01 \mathrm{~mm}$ ) was used for measurement. Before each measurement, calibration of the caliper was performed. To locate the voxel-based morphometry (VBM) of individual clinical crown, two landmarks, the proximal incisal contact area position and the apical contact area position of the tooth, furnished as the reference points. Both points were joined, and the center was marked for each line. A line joining the two centers was then extended till the gingiva to establish the VBM. After locating the VBM, the highest point in the contour of the gingiva was marked to establish the GZP, and the linear distance was noted (Figs 3 and 4). This was performed on each tooth (central incisors, lateral incisors, and canines) to distinguish the location of GZP in medial and lateral direction. A tangent line was then drawn connecting the GZP of central incisor and canine, and the vertical linear distance of the GZP of the lateral incisor from this tangent was measured to establish the GZL of anterior maxillary dentition (Fig. 5).

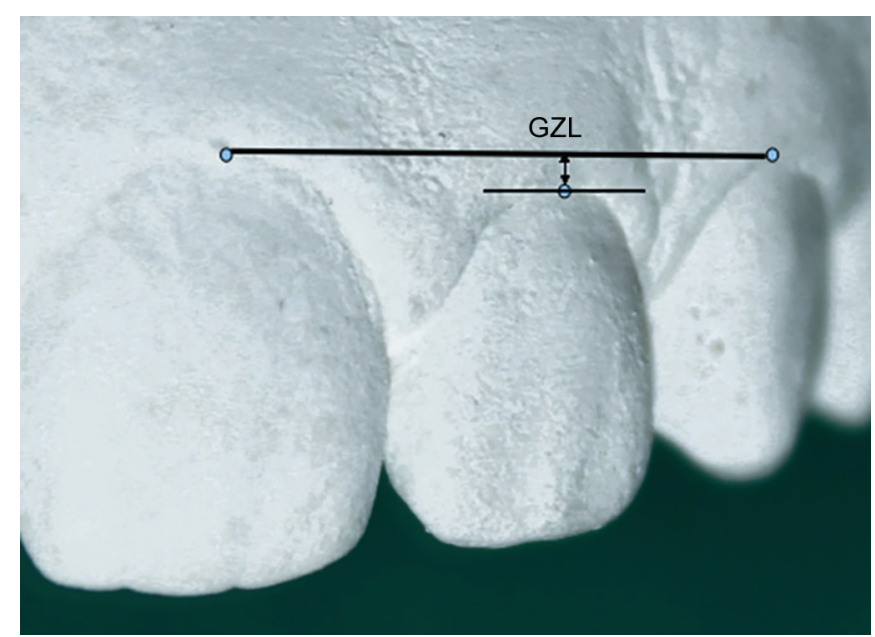

Fig. 5: Measurement of GZLs

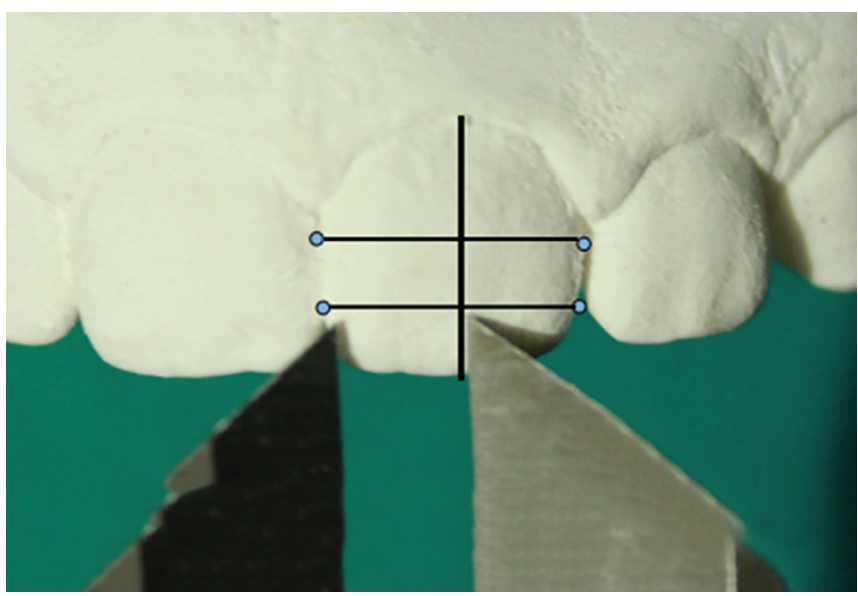

Fig. 4: Measurement of vertical bisected midline using digital caliper

\section{Statistics}

Data were analyzed using Statistical Package for the Social Sciences version 10.0/PC (SPSS Inc., Chicago, Illinois). All results are expressed as mean \pm standard deviation (SD). Intermeasurement differences were analyzed with the paired $t$-test for continuous variables and the $\chi^{2}$ test for percentages. And a value $\mathrm{p}<0.05$ was used to declare statistical significance. Finally, a multiple regression analysis was used to determine if age and sex were related to changes in GZP and GZL.

\section{Examiner Reliability}

To verify examiner reliability, all measurements were repeated on 10 randomly selected records 1 month after the original measurements. Paired t-test analysis was used to compare the original and repeated data. None of the eight measurements (GZP for 11, 12, 13, 21, 22, and 23 and the GZL for 12, 22) showed any statistical difference $(p=0.05)$ between the original and repeated values.

\section{RESULTS}

All the proclined central and lateral incisors showed a distal GZP from the VBM. There was no correlation found between age and sex when GZP and GZL were considered (Table 1). The mean GZP for central, lateral, and canine was $0.9,0.5$, and $0.20 \%$ of canine population, which showed distal GZP from VBM and rest $80 \%$ showed centralized GZP. The GZL for both the lateral incisors was found apical by approximately a mean of $0.8 \mathrm{~mm}$.

A two-sample t-test was performed, and statistically significant difference between the proclined group in regard to the location of GZP and GZL of anterior maxillary dentition was obtained for central incisors (Table 2). Highly significant results were obtained when mild proclination (25-29) was compared with severe proclination. 
GZPs and GZLs of Maxillary Anterior Dentition in Cases of Bimaxillary Protrusion

Table 1: Comparison of measurements of GZP and GZL among male and female subjects

\begin{tabular}{|c|c|c|c|}
\hline & Male & Female & \\
\hline & Mean $\pm S D$ & Mean $\pm S D$ & $p$-value \\
\hline GZP for 11 & $1.03 \pm 0.24$ & $0.98 \pm 0.21$ & 0.62 \\
\hline GZP for 21 & $1.00 \pm 0.20$ & $0.85 \pm 0.15$ & 0.07 \\
\hline GZP for 12 & $0.61 \pm 0.15$ & $0.53 \pm 0.18$ & 0.30 \\
\hline GZP for 22 & $0.56 \pm 0.17$ & $0.52 \pm 0.17$ & 0.61 \\
\hline GZP for 13 & $0.09 \pm 0.15$ & $0.08 \pm 0.19$ & 0.92 \\
\hline GZP for 23 & $0.10 \pm 0.12$ & $0.08 \pm 0.17$ & 0.75 \\
\hline GZL for 11 & $0.76 \pm 0.13$ & $0.91 \pm 0.31$ & 0.25 \\
\hline GZL for 22 & $0.74 \pm 0.11$ & $0.83 \pm 0.28$ & 0.45 \\
\hline
\end{tabular}

\section{DISCUSSION}

The perspective of gingiva is concerned with soft tissue encasing of the teeth; the basic agenda of this research is to discuss the variability of anterior dental esthetics citing physiological norms which are supposed to be helpful for clinicians to assess the treatment modality. The dentogingival esthetic units have been studied extensively in dental literature. Rufenacht in his study suggested that for a class 1 occlusion, the ideal GZL should be where the gingival contours of the central incisors and canines are at the same level and the lateral incisor positioned slightly more coronal. Though having a sample of class 1 malocclusion but with varying crown inclination, we concluded the same as Rufenacht. ${ }^{17} \mathrm{He}$ also concluded in class 2, division 2 malocclusions, the GZL of the lateral incisors is more apical compared with that of the central incisors and canines, as the laterals tend to overlap the distal aspects of the central incisors.

Quality has many expressions but indeed targeting perfection is its own reward for proper rehabilitation of anterior dental esthetics; an adequate width-length ratio of the teeth along with the GZP are most enticing characteristics aiming to restoring a proper smile. Quantitative and qualitative evaluation of the degree of crown inclination and position of the GZP and levels has not yet been extensively studied. With growing modernization, knowledge about the position of gingival complex would enable us to perform evidence-based orthodontics and achieve the desired result, which fulfills the need of high standard esthetics in this scenario. This study provided us an opportunity to assess the esthetic principle and its conduct in interdisciplinary esthetic rehabilitation. Descriptive analysis of the measurements of proclination ratio revealed distinctiveness in each group with respect to its esthetic principle. Although the position of the zenith of the gingival tissue seems like a small detail, it can greatly influence the axial inclination and emergence profile of the teeth. The crown inclination and crown angulation tend to play a seminal role
Table 2: Intergroup comparison of various proclination

\begin{tabular}{llll}
\hline & $V 1$ & $V 2$ & Significant \\
\hline GZP for 11 & $25-29$ & $30-34$ & $0.042^{*}$ \\
& $35-39$ & $0.016^{*}$ \\
& $40-44$ & $0.048^{*}$ \\
& $45-50$ & 0.099 \\
& $30-34$ & $35-39$ & 0.534 \\
& $40-44$ & 0.870 \\
& $45-50$ & 0.906 \\
& $35-39$ & $30-34$ & 0.534 \\
& $40-44$ & 0.419 \\
& $45-50$ & 0.557 \\
& $40-44$ & $45-50$ & 1.000 \\
\hline
\end{tabular}

*Mean difference is significant at 0.05 level

in the positioning of the tooth as well as its supporting structures. In our study, we aimed to decipher the effect of the crown inclination on the contour of its supporting gingiva. From Table 1, we conclude that the GZP for central incisors in males is more distal as compared with GZP of central incisors in females. When intergroup correlation of various proclination was done, we found that with an increase in proclination there was a certain change in location of GZP. The multiple regression analysis performed in our study showed no correlation of age and sex with the location of GZP and GZL. There is no consequence literature available for reference comparing GZP and crown inclination.

It has been postulated that excessive proclination of incisors might be attributed to gingival recession as the unbalanced tooth arch relationship results in buccally prominent teeth enclosed by a thin or nonexistent labial plate of bone and inadequate keratinized gingiva. ${ }^{18}$ As per the result of our study, excessive proclination does affect the position of gingival zenith where it might alter its distance from the vertical bisected midline. Statistically significant results were found when the intergroup correlation was done. As the present research has crosssectional design of the study, which only collected data at a single point of time, associations identified in this study should be interpreted with caution. Further, it must be stressed data being collected at a single point only sets a baseline for comparative evaluation of proclination and GZP and in no sense renders the effect of fixed orthodontic treatment on the gingival contour. On comparing the data obtained from the contralateral sides of the arch, all patients were found to be considerably symmetrical despite the variation in proclination. Both groups presented with values that were very close, and for the mild variation it could be inferred that there might be a very minute discrepancy between the mesiodistal dimensions for the six anterior teeth which generally goes unnoticed, however. Clinicians must take other clinical parameters 
into consideration, both in planning and in the final stage of their clinical cases.

One weakness of our study would be inability to quantify the extent of the effect of proclination varying the exact shift in the location of GZP. In our study, we relied on the measurements from cast rather than clinical examination. Trentini et $\mathrm{al}^{19}$ in their study stated no statistically significant difference between measurement obtained from cast and clinical examination; however, questions can be raised as data collected from models are inconsistent and prone to iatrogenic errors. Finally, to give a broad conclusion with no large pool of data sets is another limitation for our study.

In our venture to probe for dentogingival esthetic complex in malocclusion cases, we intend to carry further research comparing various other malocclusion patterns and setting a baseline for gingival contour followed by fixed orthodontic therapy.

\section{CONCLUSION}

This study provided direct quantitative measurements and qualitative descriptive statistics of the relative position of the GZP and GZL in correspondence to varying crown inclination. Our finding highlights the prime importance of achieving and maintaining proper gingival architecture during orthodontic treatment.

\section{CLINICAL SIGNIFICANCE}

Orthodontics is essential in the multidisciplinary team for the treatment of patients with bimaxillary protrusion, as these procedures are necessary to restore the shape and proportionality of the smile and contribute to both functional and esthetic reestablishment of the patient. ${ }^{20,21}$

\section{REFERENCES}

1. Calvin, SC. Dental orthopedia and correction of cleft palate. Chicago (IL): C. S. Case Company; 1921.

2. Scott $\mathrm{SH}$, Johnston LE. The perceived impact of extraction and nonextraction treatments on matched samples of African American patients. Am J Orthod Dentofacial Orthop 1999 Sep;116(3):352-358.

3. Farrow AL, Zarrinnia K, Azizi K. Bimaxillary protrusion in black Americans-an esthetic evaluation and the treatment considerations. Am J Orthod Dentofacial Orthop 1993 Sep;104(3):240-250.

4. Fonseca RJ, Klein WD. A cephalometric evaluation of American Negro women. Am J Orthod 1978 Feb;73(2):152-160.
5. Rosa RA, Arvystas BA. An epidemiologic survey of malocclusions among American Negroes and American Hispanics. Am J Orthod Dentofacial Orthop 1978 Mar;73(3):258-273.

6. Lew K. Profile changes following orthodontic treatment of bimaxillary protrusion in adults with the Begg appliance. Eur J Orthod 1989 Nov;11(4):375-381.

7. Tan TJ. Profile changes following orthodontic correction of bimaxillary protrusion with a preadjusted edgewise appliance. Int J Adult Orthodont Orthognath Surg 1996 Feb;11(3):239-251.

8. Lamberton CM, Reichart PA, Triratananimit P. Bimaxillary protrusion as a pathologic problem in the Thai. Am J Orthod 1980 Mar;77(3):320-329.

9. Magne, P.; Belser, U. Bonded porcelain restorations in the anterior dentiton. A biomimetic approach. Carol Stream (IL): Quintessence; 2002. p. 58-64.

10. Loe, H.; Listgarten, MA.; Terranova, VP. The gingiva: Structure and function. In: Genco RJ, Goldman HM, Cohen DW, editors. Contemparary periodontics. St. Louis (MO): C.V. Mosby; 1990. p. 3-32.

11. Rufenacht, C. Fundamentals of esthetics. Chicago (IL): Quintessence Publishing; 1990. p. 124-127.

12. Jones, L. Predictable "Gum Lifts" made easy. Oral Health and Dental Practice Management. 2003. [cited 2007 Nov 24]. Available from: http:/ / www.oralhealthjournal.com/issues / ISarticle.asp?id=97041\&story_id $=155240115507 \&$ issue $=04012$ $003 \& P C=$.

13. Goodlin, R. Gingival aesthetics: a critical factor in smile design. Oral Health Dent Pract Manage. 2003. [cited 2007 Nov 24]. Available from: http:/ / www.oralhealthjournal.com/issues/ ISarticle.asp?id=97035\&story_id $=155216115410 \&$ issue $=04012$ $003 \& P C=$.

14. Smallwood, TW. Contemporary esthetics platinum paradigm. Phoenix (AZ): Visual Smile Creations; 2003. p. 2-20.

15. Proffit, W. Contemporary orthodontics. St Louis (MO): CV Mosby Company; 1986. p. 121-129.

16. Chu SJ, Tan JH, Stappert CF, Tarnow DP. Gingival zenith positions and levels of the maxillary anterior dentition. J Esthet Restor Dent 2009 Apr;21(2):113-120.

17. Rufenacht, CR. Principles of esthetic integration. Chicago (IL): Quintessence; 2000. p. 154.

18. Hamp SE, Lundström F, Nyman S. Periodontal conditions in adolescents subjected to multiband orthodontic treatment with controlled oral hygiene. Eur J Orthod 1982 May;4(2):77-86.

19. Trentini CM, Moriarty JD, Phillips C, Tulloch JF. Evaluation of the use of orthodontic records to measure the width of keratinized tissue. J Periodontol 1995 Jun;66(6):438-442.

20. Sunguino R, Furquim LZ. Uma abordagem estética e funcional do tratamento ortodôntico em pacientes com agenesias de incisivos laterais superiores. Rev Dent Press Ortodon Ortopedi Facial 2003 Nov-Dec;8(6):119-157.

21. Zachrisson BU. Improving the esthetic outcome of canine substitution for missing maxillary lateral incisors. World J Orthod 2007 Feb;8(1):72-79. 